\title{
Development of an atom buncher
}

Cite as: Journal of Applied Physics 55, 1278 (1984); https://doi.org/10.1063/1.333216

Submitted: 19 August 1983 . Accepted: 01 November 1983 . Published Online: 04 June 1998

G. S. Hurst, M. G. Payne, R. C. Phillips, et al.

\section{Challenge us.}

What are your needs for periodic signal detection?

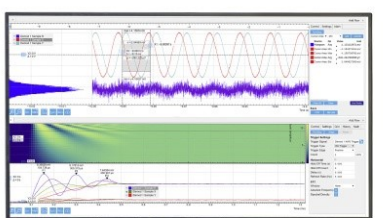

Zurich

Instruments 


\section{Development of an atom buncher}

G. S. Hurst, M. G. Payne, and R. C. Phillips

Chemical Physics Section, Health and Safety Research Division, Oak Ridge National Laboratory, Oak Ridge, Tennessee 37830

J.W.T. Dabbs

Engineering Physics Division, Oak Ridge National Laboratory, Oak Ridge, Tennessee 37830

B. E. Lehmann

University of Bern, Bern, Switzerland

(Received 19 August 1983; accepted for publication 1 November 1983)

An "atom buncher" for controlling the concentration of gaseous samples has been conceptualized, evaluated theoretically, fabricated, and tested with excellent results. In effect, the atom buncher greatly increases the probability that a free atom will be in a small detector volume at a desired time. This was accomplished by using cryogenic techniques to condense atoms on a small spot and a pulsed laser to momentarily heat the spot to release the atoms at the desired time. Our work on noble gas atom counting by using resonance ionization spectroscopy is discussed as one example of the applications of the atom buncher.

PACS numbers: 35.10.By, 51.90. + r, 07.20.Mc

\section{INTRODUCTION}

In the process of developing a Maxwell demon ${ }^{1}$ having the capability of counting a few noble gas atoms we had to develop an "atom buncher." The need for an atom buncher arose from the fact that some of the applications ${ }^{2}$ of Maxwell's demon require counting 10-100 atoms of a particular isotope which are free to move in a 4-liter vacuum enclosure, yet our laser scheme which ionizes the atoms [in the Resonance Ionization Spectroscopy (RIS) process ${ }^{3}$ ] sweeps out a volume of less than $10^{-3} \mathrm{~cm}^{3}$ per laser pulse. In this process an unacceptably large number $\left(>10^{7}\right)$ of laser shots would be required to ionize and count all of the atoms. Thus, we conceived of an atom buncher which would put a large fraction of the target atoms in the laser beam at the time of the shot, making the probability of ionizing and counting a given atom $10 \%$ or greater.

Applications of isotopically selective atom counting could include solar neutrino measurements $\left({ }^{81} \mathrm{Kr}\right), \beta-\beta$ decay $\left({ }^{82} \mathrm{Kr},{ }^{128} \mathrm{Xe},{ }^{130} \mathrm{Xe}\right)$, ground water dating $\left({ }^{81} \mathrm{Kr}\right)$, polar ice cap dating $\left({ }^{81} \mathrm{Kr}\right)$, and oceanography $\left({ }^{39} \mathrm{Ar}\right)$. The atom buncher is a key part of the apparatus for counting atoms for these and other applications. Furthermore, there are many other uses of a device for producing space and time correlations of free atoms and molecules. For instance, small clouds of a noble gas could be isolated in a vacuum system for more effective vacuum ultraviolet (VUV) generation with lasers, and small samples could be released into a vacuum for spectroscopic studies in applications similar to those of the pulsed nozzle. This paper describes, therefore, the atom buncher in a general way to make it more accessible to these wider applications.

\section{CONCEPT OF AN ATOM BUNCHER}

The basic idea of the atom buncher is very simple, as shown in Fig. 1. A cold surface is used to condense the atoms of interest and a pulse of laser light momentarily heats a thin layer of the cold surface. Atoms are evaporated as a conse- quence of the laser heating and pass through a region of space just above the cold surface (the detector volume in Fig. 1) after a short time of flight. Thus, there is a high probability that the atoms will be in the detector volume at the desired time. Long before atoms can return by random walk the surface is again cold enough to condense them.

Physical adsorption of noble gases on cooled surfaces can be characterized by the concept of mean stay times. ${ }^{4}$ Values for $\tau_{0}$ and $\epsilon$ for several noble gas/metal systems have been measured, where $\tau_{0}$ is the preexponential factor and $\epsilon$ is the heat of adsorption in the expression for the mean stay time $\tau$ on a surface with temperature $T_{s}$ :

$$
\tau=\tau_{0} e^{\epsilon / k T_{s}}
$$

Values for xenon and krypton on copper and nickel surfaces

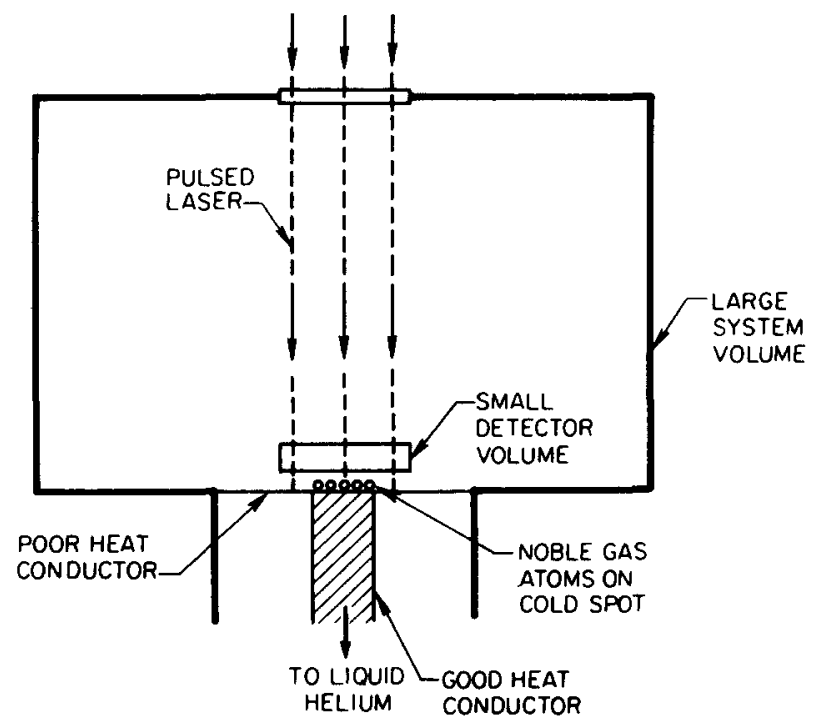

FIG. 1. Concept of the atom buncher. In the steady state, noble gas atorns are condensed on a cold spot which can be heated in its entirety with a pulsed laser beam. Atoms are quickly released and are found in a small detector volume within a few microseconds after the laser is pulsed. 
ranged from $10^{-15}$ to $10^{-20} \mathrm{sec}$ for $\tau_{0}$ and from 0.22 to 0.28 $\mathrm{eV}$ for $\epsilon$, depending on the surface treatment of the samples that were studied. We define a critical temperature $T_{c}$ as the temperature where the mean stay time $\tau$ is $1 \mu \mathrm{sec}$. From the data published, ${ }^{4}$ one estimates that $T_{c} \cong 110 \mathrm{~K}$ for krypton and $T_{c} \cong 130 \mathrm{~K}$ for xenon, the exact value depending on the specific surface conditions. If a laser pulse can heat a surface ${ }^{5}$ and keep $T>T_{c}$ for several microseconds, the whole noble gas sample, which is initially trapped on the cold surface, will leave the surface. In fact, it would be difficult to control $T_{c}$ well enough to have only a fraction of the atoms leave the cold tip; essentially it is all or nothing.

Recurrence times to a small area $A$ in an open system of volume $\mathrm{V}$ are approximately

$$
t_{R}=\frac{4 V}{A \bar{v} \epsilon},
$$

where $\bar{v}$ is the mean speed of the free atoms and $\epsilon$ is the sticking probability. This recurrence time determines the number of atoms $N$ in the free state at time $t$ according to

$$
N=N_{0} e^{-t / t_{R}},
$$

where $N_{0}$ is the number of free atoms at $t=0$. (The single exponential behavior assumes that all apertures in the vacuum system have an area which is large compared with that of the atom buncher.) Even where $V$ is one liter and $A$ is a few $(\mathrm{mm})^{2}, t_{R}$ can be as small as a few seconds; thus, noble gas atoms can be recycled reasonably rapidly. In our own application where lasers are used for resonance ionization of noble gases, it is not necessary, or even desirable, that all of the atoms be on the cold spot when the heating laser is pulsed. This is consistent with digital counting of individual atoms.

To clarify the need for an atom buncher we discuss our own application in more detail. We are interested in counting each atom in a small sample of a noble gas, e.g., a few hundred ${ }^{81} \mathrm{Kr}$ atoms for solar neutrino and groundwater dating applications. ${ }^{2}$ It turns out that the volume $(V)$ of an apparatus required so that a mass spectrometer can be used to provide isotopic resolution is about 4 liter. On the other hand, the laser system ${ }^{6}$ for generating VUV radiation at $1165 \AA$ for one-photon excitation (the first step of resonance ionization) of krypton will saturate a volume $(\Delta V)$ of only about $4 \times 10^{-4} \mathrm{~cm}^{3}$. The probability of counting in this incoherent case is $P_{i}=\Delta V / V$. To detect $95 \%$ would require nearly $3 \times 10^{7}$ laser pulses or $833 \mathrm{~h}$ with a system that pulsed at 10 times per second. In the coherent case the probability is $P_{c}(t)=P_{b} P_{I}(\Delta t)$, where $P_{b}$ is the probability that an atom is on the buncher and $P_{I}(\Delta t)$ is the probability that an atom leaving the cold tip is in the laser volume $\Delta V$ after the laser delay $\Delta t$. Suppose the laser volume $\Delta V$ is defined by a laser beam that is about $0.05 \mathrm{~cm}$ in diameter and projects $0.2 \mathrm{~cm}$ of length onto a collection aperture; then $\Delta V=4 \times 10^{-4} \mathrm{~cm}^{3}$. If the small detector volume is centered about $1 \mathrm{~mm}$ from the cold tip, it takes about $10 \mu \mathrm{sec}$ for krypton atoms (at $120 \mathrm{~K}$ ) to fill it if the atoms have a mean velocity distribution characteristic or release at $T=120 \mathrm{~K}$. In this case $P_{I}(\Delta t=10$ $\mu \mathrm{sec})$ can be as large as $2 \%$. Therefore, $P_{c}(t)=0.02 t_{L} / t_{R}$, where $t_{L}$ is the laser repetition time and is small compared to the recurrence time $t_{R}$. Assuming that most of the atoms are free, we have $P_{c}>P_{i}$ when $t>(\Delta V / V) 10 t_{R}$. For example, if $\Delta V / V=10^{-7}, t_{R}=10 \mathrm{sec}$, and $t=0.1 \mathrm{sec}$, we have $P_{c}=2 \times 10^{-4}$ compared to $P_{i}=10^{-7}$. For a lower laser repetition rate, the enhancement of $P_{c}$ over $P_{i}$ is even more dramatic; $P_{c}$ approaches $2 \%$ when $t_{L}$ is long compared to $t_{R}$.

\section{THEORETICAL CONSIDERATIONS}

In this section we present simple theoretical considerations that were used for the design of a buncher with characteristics chosen to suit the needs inferred in the Introduction. The compatibility of the design with the construction and durability of the device will be discussed in the next section.

\section{A. Calculation of the steady-state temperature profile}

We first consider the calculation of the temperature profile for the tip of the cold finger shown in the design of Fig. 2. In the absence of radiative heating and in the steady state, the total heat flow down the nickel rod to the liquid helium is the same as that down the 304 stainless steel cylinder. Let this constant heat current be $I$ and define the thermal conductivity $\kappa(T)$ through $\bar{J}=-\kappa(T) \bar{\nabla} T$, where $\bar{J}$ is the heat current density and $T$ is the absolute temperature. If we define $U_{\mathrm{Ni}}(T)=\int_{0}^{T} \kappa_{\mathrm{Ni}}\left(T^{\prime}\right) d T^{\prime}$ for the nickel rod, then

$$
U_{\mathrm{Ni}}\left(T_{1}\right)-U_{\mathrm{Ni}}\left(T_{0}\right)=+\frac{I L_{1}}{\pi R_{0}^{2}},
$$

where $L_{1}$ is the distance from the liquid helium reservoir to the nickel disk, $R_{0}$ is the radius of the nickel rod, $\kappa_{\mathrm{Ni}}(T)$ is the thermal conductivity of the nickel rod, $T_{0}$ is the temperature at the liquid helium reservoir, $T_{1}$ is the temperature of the nickel where it joins the stainless steel disk, and $I$ is the total heat current, assumed constant.

Further, let $T_{2}$ be the temperature of the stainless steel disk where it joins the outer 304 stainless steel jacket, $R_{1}$ the radius of the stainless steel disk, $U_{s}(T)=\int_{0}^{T} \kappa_{s}\left(T^{\prime}\right) d T^{\prime}, \kappa_{s}(T)$ the thermal conductivity of stainless steel, $\mu_{1}$ the thickness of stainless disk, and $\mu_{2}$ the thickness of the stainless steel jacket.

Considering the thin stainless disk, we find

$$
U_{s}(T)-U_{s}\left(T_{1}\right)=\frac{I}{2 \pi \mu_{1}} \ln \left[\frac{R}{R_{0}}\right] .
$$

The logarithm arises due to the requirement that in the disk $J(\rho) 2 \pi \rho \mu_{1}=I=$ constant. We now consider the outer jacket with length $L_{2}$ :

$$
U_{s}\left(T_{3}\right)-U_{s}\left(T_{2}\right)=\frac{I L_{2}}{2 \pi R_{1} \mu_{2}},
$$

where $T_{3}=290 \mathrm{~K}$ and $L_{2}$ extends from the disk to the point where the thickness of the stainless becomes much greater (i.e., $L_{2}=25.4 \mathrm{~mm}$ ) and is thus a heat sink at room temperature. Therefore,

$$
\begin{aligned}
U_{s}\left(T_{2}\right)-U_{s}\left(T_{1}\right) & =\frac{I}{2 \pi \mu_{1}} \ln \left[\frac{R_{1}}{R_{0}}\right] \\
& =U_{s}\left(T_{3}\right)-U_{s}\left(T_{1}\right)-\frac{I L_{2}}{2 \pi R_{1} \mu_{2}} .
\end{aligned}
$$




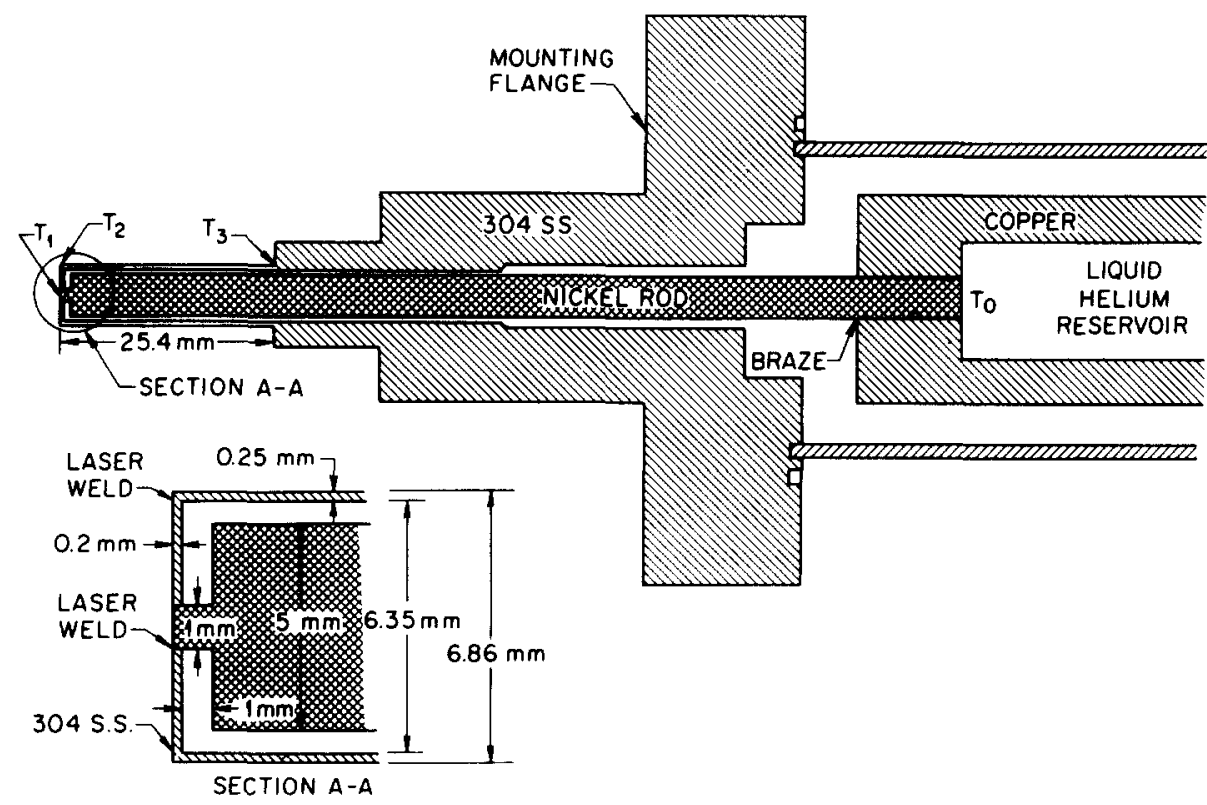

FIG. 2. Schematic diagram and some construction details for the cold tip portion of the atom buncher.

Using Eqs. (4) and (7), we get

$$
\begin{aligned}
U_{s}\left(T_{3}\right)-U_{s}\left(T_{1}\right)= & {\left[U_{\mathrm{Ni}}\left(T_{1}\right)-U_{\mathrm{Ni}}\left(T_{0}\right)\right] \frac{\pi R_{0}^{2}}{L} } \\
& \times\left[\frac{L_{2}}{2 \pi R_{1} \mu_{2}}+\frac{1}{2 \pi \mu_{1}} \ln \left(\frac{R_{1}}{R_{0}}\right)\right] .
\end{aligned}
$$

Since $T_{3}=290 \mathrm{~K}$ and $\tau_{0} \simeq 4.2 \mathrm{~K}$, the only unknown in Eq. (5)

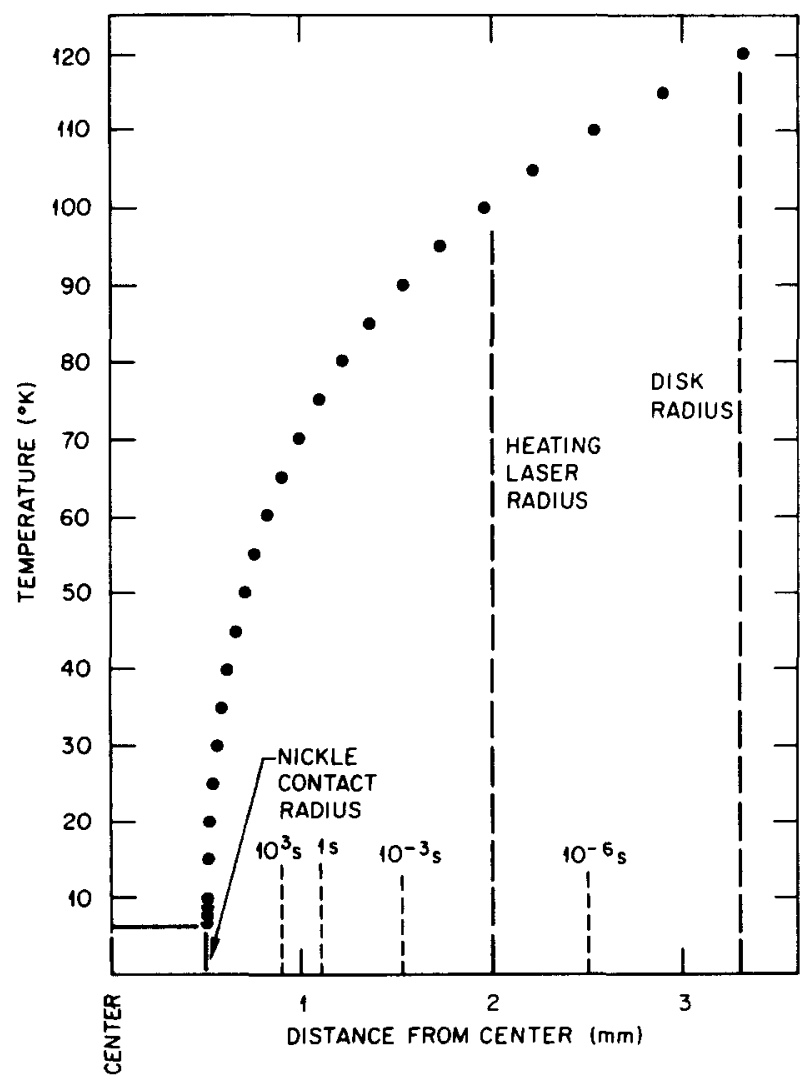

FIG. 3. Calculations of the temperature profile of the buncher tip for the steady state and some representative stay times for krypton atoms at various distances from the center of the disk. is $T_{1}$. Thus, if we fit polynomials to $\kappa_{s}$ and $\kappa_{\mathrm{Ni}}$

$$
\begin{aligned}
& \kappa(T)=\sum_{n=0}^{N} C_{n} T^{n}, \\
& U(T)=\sum_{n=0}^{N} \frac{C_{n} T^{n+1}}{n+1},
\end{aligned}
$$

we can find the real positive root of the polynomial in Eq. (8) for the value of $T_{1}$. Using actual data for fitting $\kappa$ for nickel ${ }^{7}$ and stainless steel ${ }^{8}$ with a fifth-order polynomial, we find $T_{1}=7 \mathrm{~K}$. Once $T_{1}$ is determined, the heat current can be determined from Eq. (4). With both $T_{1}$ and $I$ now known we can use Eq. (5) to determine $R$ values on the disk at which a temperature $T$ occurs, i.e.,

$$
R(T)=R_{0} \exp \left\{\frac{2 \pi \mu_{1}}{I}\left[U_{s}(T)-U_{s}\left(T_{1}\right)\right]\right\} .
$$

The calculated temperature profile for the design of Fig. 2 is shown in Fig. 3. Representative stay times are shown at several values of the distance from the center of the buncher. The radius of the heating laser beam must be made large enough so that the stay time at that radius is not so long that atoms may "hide" from the pulsed laser beam. The meaning of the term "hide" depends on the application of the atom buncher.

\section{B. Calculations of the laser heating transient}

We now discuss the characteristics of a pulsed laserinduced temperature transient for the actual design of Fig. 2 to determine the conditions required for the release of krypton atoms from the cold surface.

When light at about 6000 - $\AA$ wavelength is incident on nickel or stainless steel, about $60 \%$ is reflected; the other $40 \%$ is absorbed within $\sim 200 \AA$ of the surface. We first consider the heating of the nickel surface since it has a higher thermal conductivity and is therefore harder to heat with the $\sim 1.4 \mu \mathrm{sec}$ laser pulses to be used experimentally. The nickel center of the cold spot has a diameter of $1 \mathrm{~mm}$; and since the laser light is absorbed near the surface, a one-dimensional 
treatment of the heat transport problem is appropriate. We need to solve the equation

$$
\frac{\partial \theta}{\partial t}=\frac{1}{\rho C_{v}(T)}\left\{\frac{\partial}{\partial x}\left[\kappa(T) \frac{\partial \theta}{\partial x}\right]+S(x, t)\right\},
$$

where $\kappa(T)$ is the thermal conductivity, $C_{v}(T)$ is the specific heat, $\rho$ is the density, $\theta=T-T_{1}$, $T$ is the absolute temperature at $x$ and at time $t, T_{1}$ is the absolute temperature before the laser pulse, independent of $x$ and $t$, and $S(x, t)$ is the amount of heat absorbed per unit time per unit volume at $(x, t)$. form

Initially, $T_{1}$ is about $7 \mathrm{~K}$ and in this region $C_{v}$ is of the

$$
C_{v}=\frac{12 \pi^{4}}{5} \frac{R}{M}\left(T / \theta_{0}\right)^{3}+\gamma T,
$$

where $M$ is the atomic weight, $R$ is the ideal gas constant, and $\theta_{0}$ is the Debye temperature. The $T^{3}$ term is due to lattice vibrations (harmonic) while the $\gamma T$ term is the sum of the electronic and the anharmonic vibrational contribution to specific heat. For nickel, $\theta_{0} \simeq 420 \mathrm{~K}$. The above functional form of $C_{v}$ is appropriate for nickel at temperatures below $150 \mathrm{~K}$.

The thermal conductivity of nickel at low temperature is very sensitive to impurities. The high purity (99.998\%) sample used in our design has

$$
\kappa(T) \simeq 2.3 \times 10^{6}\left[\mathrm{erg} / \mathrm{sec} \mathrm{K}^{2} \mathrm{~cm}\right] T,
$$

for $0.4 \mathrm{~K}<T<10 \mathrm{~K}$. Between 10 and $100 \mathrm{~K},(T)$ varies between $2 \times 10^{7} \mathrm{erg} / \mathrm{sec} \mathrm{K} \mathrm{cm}$ and $4 \times 10^{7} \mathrm{erg} / \mathrm{sec} \mathrm{K} \mathrm{cm} \mathrm{with}$ the maximum conductivity occurring at $T \sim 30 \mathrm{~K}$. Nickel exhibits typical metal behavior, having temperature independent $\kappa(T)$ above $\theta_{0}$.

We see that both $C_{v}(T)$ and $\kappa(T)$ exhibit rather sharp temperature dependences for $7 \mathrm{~K}<T<150 \mathrm{~K}$, making the heat flow equation nonlinear and thereby excluding the possibility of an exact analytical solution. The very thermal properties that made it possible to construct the atom buncher by welding techniques complicate the understanding of the laser heating transient.

We now discuss explicitly the laser heating of a nickel surface. We first note that at very low temperature $\rho C_{v}$ is very small and that the diffusiveness $D=\kappa(T) / \rho C_{v}(T)$ is correspondingly large. Thus, the absorbed heat initially spreads out very quickly with $\bar{x}^{2} \simeq \int_{0}^{t}\left\{\kappa\left[T\left(t^{\prime}\right)\right] / \rho C_{v}\left[T\left(t^{\prime}\right)\right]\right\} d t^{\prime}$, where $\bar{x}^{2}(t)$ is the mean squared depth over which the heat is spread. However, with a very small $C_{v}(T)$ it takes very little heat per unit volume to cause a significant temperature rise; thus, in spite of rapid diffusion, the temperature at the surface rises rapidly during the very early part of the laser pulse. Also, the peak temperature (say $120 \mathrm{~K}$ ) is quite insensitive to the initial temperature (say 7-20 K).

In the region between 20 and $150 \mathrm{~K}$ no serious error is made if we use $\rho C_{v}=A T^{3}$, and $\kappa(T)=\kappa_{0}=$ constant $=2 \times 10^{7} \mathrm{erg} / \mathrm{cm} \mathrm{sec} \mathrm{K}$. With these simplified forms for $\kappa(T)$ and $\rho C_{v}(T)$ the heat flow equation becomes

$$
A T^{3} \frac{\partial \theta}{\partial t}=\kappa_{0} \frac{\partial^{2} \theta}{\partial x^{2}}+\frac{Q_{0}}{\tau} S(t / \tau) \delta(x), x \geqslant 0 .
$$

Above, we assume that the energy is absorbed in a very thin layer near the surface and that the laser pulse shape is given by $S(t / \tau)$, where $\tau$ is a measure of the pulse length, $Q_{0}$ is the total absorbed energy per unit area, and

$$
\int_{0}^{\infty} S(w) d w=1 .
$$

Noting that $T_{1}$ is constant we change to dimensionless variables $u, v, w$ where

$$
\begin{aligned}
& T=\left(Q_{0}^{2} / \kappa_{0} \tau A\right)^{1 / 5} u(w, v), \\
& t=\tau w, \\
& x=\left(\kappa_{0}^{4} \tau^{4} / A Q_{0}^{3}\right)^{1 / 5} v .
\end{aligned}
$$

The heat flow equation becomes

$$
\frac{\partial u}{\partial w}=\frac{1}{u^{3}} \frac{\partial^{2} u}{\partial v^{2}}+\frac{1}{u^{3}} S(w) \delta(v),
$$

where $u$ is to be solved for subject to the conditions

$$
\begin{aligned}
u(0, v) & =u(\infty, v)=u(w, \infty)=\left(\kappa_{0} \tau A / Q_{0}^{2}\right)^{1 / 5} T_{1} \\
& =\left(\kappa_{0} \tau A T_{1}^{5} / Q_{0}^{2}\right)^{1 / 5} .
\end{aligned}
$$

The numerical studies of $T$ show that the maximum temperature attained, $T_{m}$, is independent of $T_{1}$ as long as $T_{m} / T_{1}>1$, and this suggests that the solution for $u$ is independent of $u(0, v)$ as long as $u(0, v)<1$. In the same way, as long as $\kappa_{0}, \tau, A, T_{1}$, and $Q_{0}$ are such that $u(0, v)<1$, the solution for $u(w, v)$ is also independent of these parameters. This solution for $u(w, v)$ then gives the proper solution for all $Q_{0}, \tau$, and $T_{1}$ as long as they are such that the predicted $T_{m}>T_{1}$. Thus,

$$
T(x, t)=\left(Q_{0}^{2} / \kappa_{0} \tau A\right)^{1 / 5} u\left[\frac{t}{\tau},\left(A Q_{0}^{3} / \kappa_{0}^{4} \tau^{4}\right)^{1 / 5} x\right] .
$$

The function $u(w, v)$ has been studied numerically for $0.05<u(0, v)<0.3$ for the particular functional form $S(w)=w e^{-w}$. In all cases the peak $u$ is near $w \simeq 2.4$ where the value of $u(2.4,0) \sim 1.15$. A very small variation $(3 \%)$ is observed in the peak value for initial values of $u(0, v)$ anywhere in the range of study. Thus, $T_{m}$ is given by

$$
T_{m} \simeq 1.15\left(Q_{0}^{2} / \kappa_{0} \tau A\right)^{1 / 5} \text {. }
$$

The decrease in $T(0, t)$ following the peak is very slow. For instance, for $t=25 \tau$

$$
T(t)=T_{m} /\left\{1+\frac{2}{3}\left[(t / \tau)-\left(t_{m} / \tau\right)\right]\right\}^{1 / 5} \text {. }
$$

The value of $T_{m}$ is not strongly dependent on pulse shape, and the decay of $T$ following the laser pulse is only very weakly dependent on pulse shape.

Figure 4 shows $\boldsymbol{u}(w, 0)$ (i.e., proportional to the surface temperature) for the initial conditions $u(0, v)=0.1,0.2$. For nickel we use $\kappa(T)=2 \times 10^{7} \mathrm{erg} / \mathrm{cm} \mathrm{sec} \mathrm{K,} \rho C_{v}(T)=40 T^{3}$ with $C_{v}(T)=40 T^{3}$ with $\rho C_{v}$ in units of $\mathrm{erg} / \mathrm{cm}^{3} \mathrm{~K}$. For the laser parameters we use $\tau=8 \times 10^{-7} \mathrm{sec}, Q_{0}=0.284 \mathrm{~J} / \mathrm{cm}^{2}$, and find $T_{m} \simeq 120 \mathrm{~K}$. Since $T_{m} \propto Q_{0}^{2 / 5} / \tau^{1 / 5}$ the value of $T_{m}$ for other $Q_{0}$ such that $T_{m} / T_{1}>4$ is easily estimated. For instance, if $\tau=8 \times 10^{-7} \mathrm{sec}$ and $Q_{0}=0.142 \mathrm{~J} / \mathrm{cm}^{2}$, we have $T_{m}=120 \mathrm{~K} / 2^{2 / 5} \simeq 91 \mathrm{~K}$, which is about the temperature at which krypton would start to be released from the surface (see the stay times in Fig. 2).

A similar treatment can be presented for stainless steel. Stainless steel and nickel have a very similar Debye tempera- 


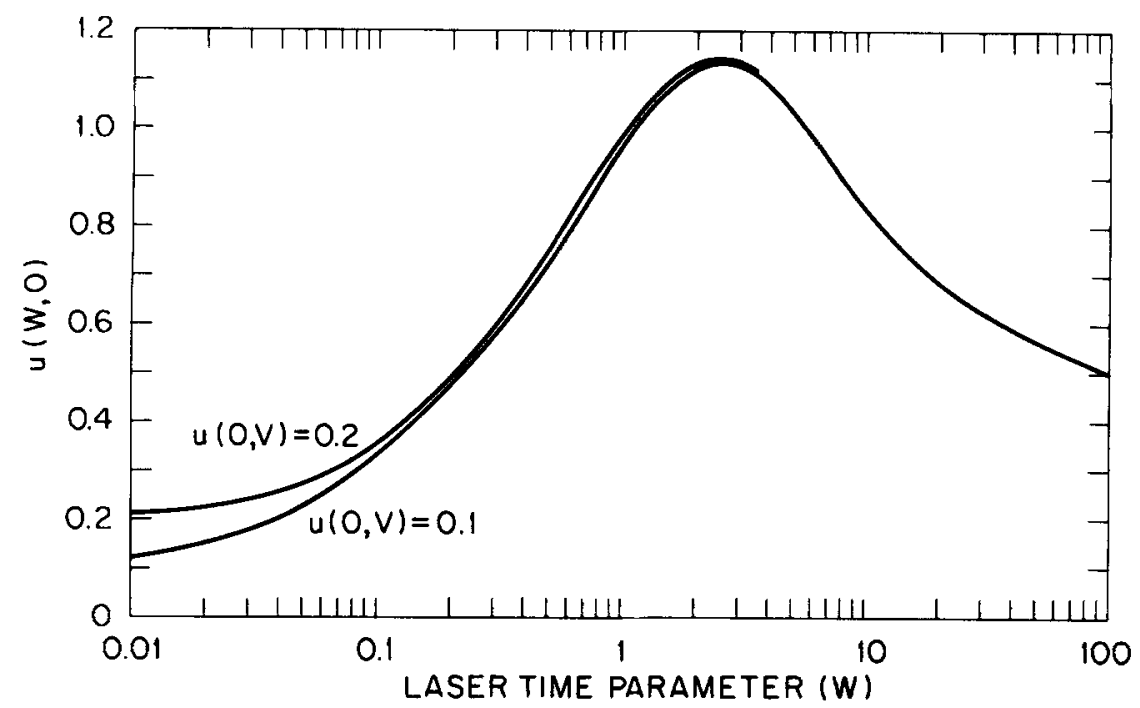

FIG. 4. Graph of $u(w, 0)$ for nickel targets, demonstrating for $u(0, v)=0.1$ and 0.2 the approximate independence of the peak height on initial conditions as long as $u(0, v)<1.15$. This curve predicts the surface temperature transient when a low temperature nickel surface is heated by the absorption of $Q_{0} \mathrm{~J} / \mathrm{cm}^{2}$ from a laser with pulse length parameter $\tau$ through the relationship $T(0, t)=\left(Q_{0}^{2} / \kappa_{0} \tau A\right)^{1 / 5} u[(t / \tau), 0]$.

ture, and for $T>20^{\circ}$ they have similar specific heats. However, $\kappa(T)$ for stainless steel is much lower than that for nickel. In particular, for $7 \mathrm{~K}<T<120 \mathrm{~K}$ we can use

$$
\kappa(T)=c T=10^{4} T
$$

for stainless steel, where $T$ is in $\mathrm{K}$ and $\kappa(T)$ is in erg/ $\mathrm{cm} \sec \mathrm{K}$. If we let

$$
T(x, t)=\left(Q_{0}^{2} / A c \tau\right)^{1 / 6} u\left[\frac{t}{\tau},\left(Q_{0} A / c^{2} \tau^{2}\right)^{1 / 3} x\right]
$$

and $u=z^{2}$, the heat flow equation becomes

$$
\frac{\partial z}{\partial w}=\frac{1}{z}\left[\left(\partial^{2} z / \partial v^{2}\right)+2 S(w) \delta(v)\right] .
$$

The insensitivity of $T_{m}$ to initial temperature for stainless steel is even more pronounced than for nickel since at low temperature the diffusiveness is not as large (for small $T$ ) in stainless steel as in nickel. Again, $u(w, v)$ will be insensitive near its peak to $u(0, v)$ if $u(0, v)<1.0$, i.e., if $T_{m} / T_{1}>1$. This is demonstrated in Fig. 5 where $u(w, 0)$ is shown for $u(0, v)=0.1$ and $u(0, v)=0.4$. Thus, for stainless steel

$$
T_{m}=1.17\left(Q_{0}^{2} / A c \tau\right)^{1 / 6} \text {. }
$$

With $C_{v}=40 T^{3}$ in cgs units, $C=1.0 \times 10^{4}$, and $\tau=8 \times 10^{-7} \mathrm{sec}$, we find that for $Q_{0}=0.142 \mathrm{~J} / \mathrm{cm}^{2}, T_{m}$ $=159 \mathrm{~K}$. As expected, stainless steel is much easier to heat than nickel. With $Q_{0}=0.0355 \mathrm{~J} / \mathrm{cm}^{2}$ (a factor of four less), we obtain $T_{m}=159 \quad \mathrm{~K} / 4^{1 / 3}=100 \mathrm{~K}$. In general $T_{m} \propto Q_{0}^{1 / 3} / \tau^{1 / 6}$. With the shorter pulse, $\tau=8 \times 10^{-9} \mathrm{sec}$, the value of $Q_{0}=0.0142 \mathrm{~J} / \mathrm{cm}^{2}$ will give a rise to $159 \mathrm{~K}$.

\section{Calculation of atoms in flight}

We have seen earlier that the stay time of krypton on metal surfaces is of the form $\tau=\tau_{0} \exp [3000 / T(K)]$, where $10^{-19} \mathrm{sec} \leqslant \tau_{0}<10^{-13} \mathrm{sec}$. Thus, the atoms are all evaporated at essentially the same time because of the rapid variation of stay time with temperature. Consider distances away from the cold surface which are small compared with the radius of the cold region (originally with $T<110 \mathrm{~K}$ ). The fraction of the released atoms between $x$ and $x+d x$ at time $\tau$ following their release is

$$
F d x=\frac{x m d x}{\tau^{2} k T_{c}} e^{-\left(m x^{2} / 2 k T_{c} \tau^{2}\right)},
$$

where $m$ is the mass of released atoms, $k$ is the Boltzman constant, and $T_{c} \simeq 110 \mathrm{~K}$. This simple expression holds if the

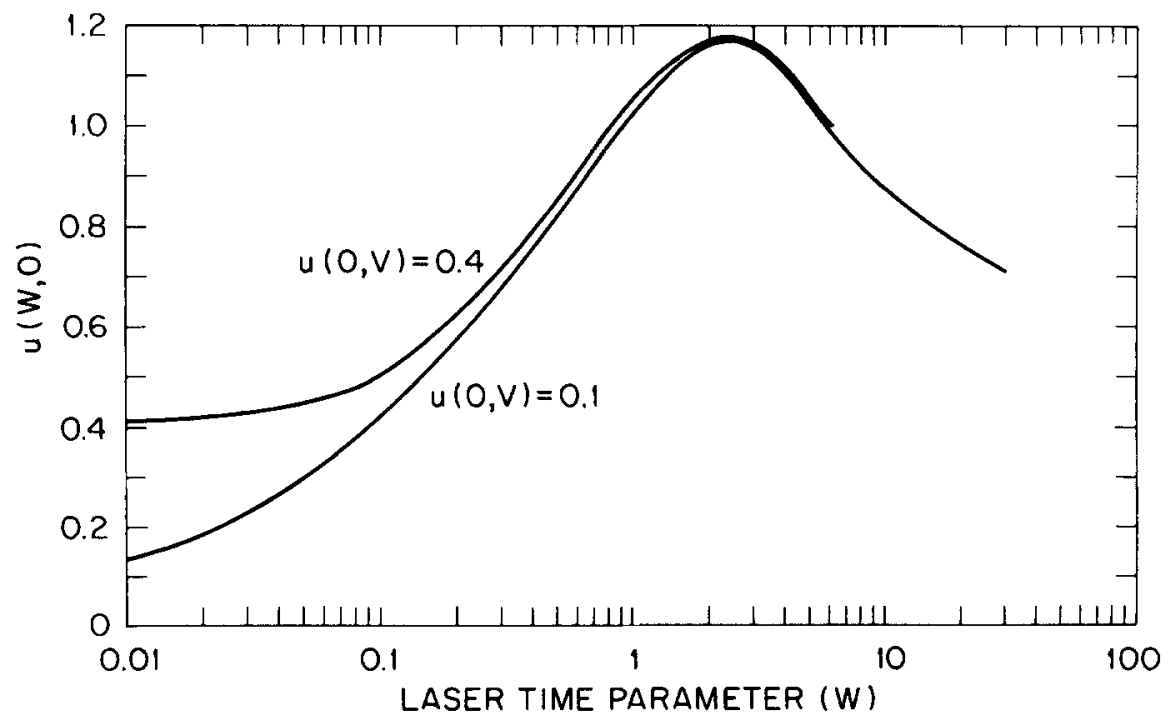

FIG. 5. Graph of $u(w, 0)$ for a stainless steel target, demonstrating for $u(0, v)=0.1$ and 0.4 the approximate independence of peak temperature on initial conditions as long as $u(0, v)<1.17$. This curve predicts the surface temperature transient when a low temperature stainless steel surface is heated by absorbing an amount of energy $Q_{0}$ $\mathrm{J} / \mathrm{cm}^{2}$ from a laser with pulse length parameter $\tau$ through the relationship $T(0, t)$ $=\left(Q_{0}^{2} / A c \tau\right)^{1 / 6} u[(t / \tau), 0]$. 


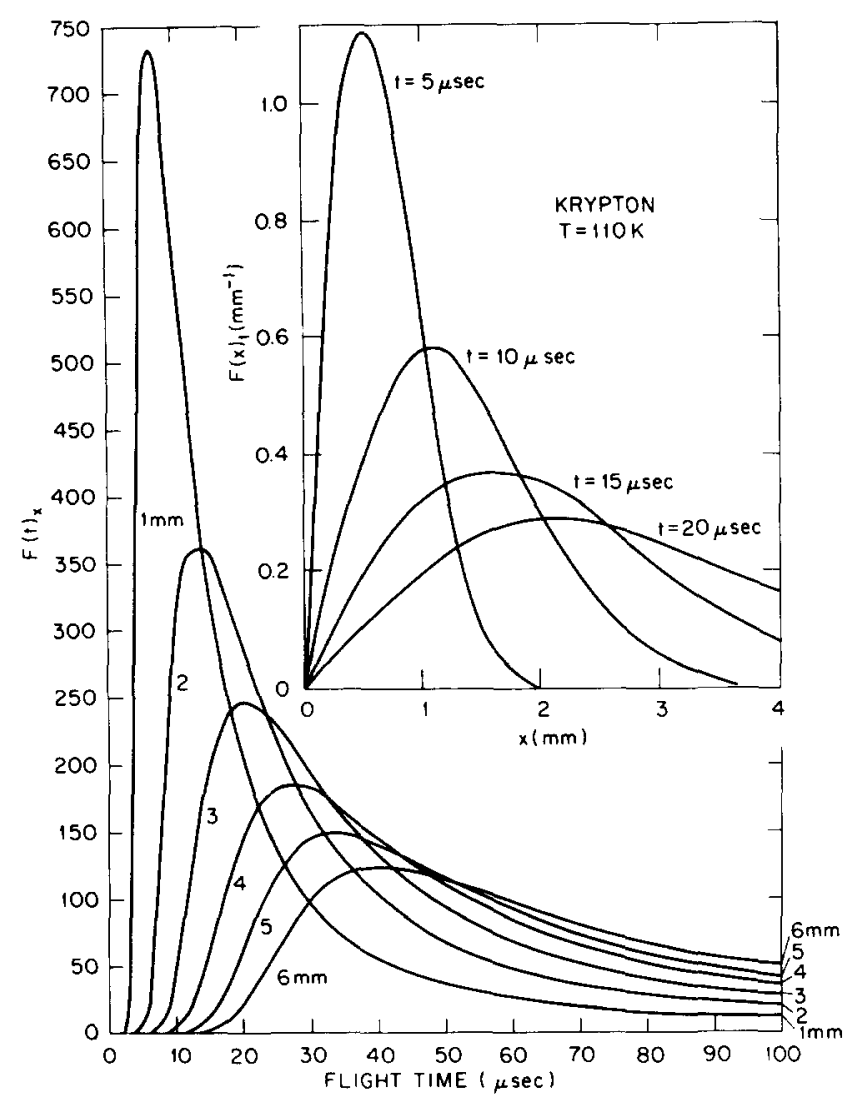

FIG. 6. Calculations of the atoms in fight from the cold finger following a heating laser pulse. The plot shows $F(t)_{x}$, the relative probability that atoms are near the indicated distance as a function of time, and $F(x)_{t}$, the spatial distribution for various delay times.

maximum temperature $T_{m}$ is at least $130 \mathrm{~K}$ over the larger part of the cold surface. In this case there is essentially no spread in the time of release (which occurs very near the time at which $T$ becomes greater than $110 \mathrm{~K}$ ).

Figure 6 shows the plot of Eq. (21) in two forms. The variation of $F$ with distance at various fixed times $F(x)_{t}$ is convenient for evaluating the probability $P_{I}(\Delta t)$ that the atoms will be ionized in the laser beam of a finite diameter at a time delay $\Delta t$. The form $F(t)_{x}$ is convenient since the delay time to maximize the signal can be quickly determined experimentally, and hence the distance of the laser beam to the buncher surface can be estimated.

\section{EXPERIMENTAL TESTS}

The atom buncher was designed for use of liquid helium as a coolant. Most of the dimensions were chosen such that the buncher could be used inside the ionization region of a quadrupole mass spectrometer. The materials were chosen according to their function in the system, as will be shown.

Coupling the liquid helium to the cryotip was accomplished by using high purity $(99.998 \%$ ) nickel. Nickel can be easily brazed to copper and it can be laser welded to stainless steel. Furthermore, the thermal conductivity of nickel is high enough that only a $3 \mathrm{~K}$ temperature rise is introduced between the liquid helium reservoir and the cryotip. Finally, nickel is easily heated by absorption of energy from a pulsed laser.

Stainless steel (304) was chosen for the thin disk material of the cryotip. This type of stainless steel is easily rolled to the desired thickness and laser welded to nickel. The low thermal conductivity of this material is suited to defining a small cold spot through large temperature gradients, as shown in the above theoretical treatment.

The resistive tube, the body, and the mounting flange were constructed from one piece of 304 stainless steel. The low thermal conductivity of the thin tube lowers the overall heat load of the system. The body is the heat source for the device, so the walls were made as thick as possible to compensate for the low thermal conductivity of stainless steel.

The remainder of the system consists of the flow lines to transport the liquid helium from a 100-liter dewar to the reservoir (which is exhausted into the room) and a vacuum shroud for insulation of the flow system.

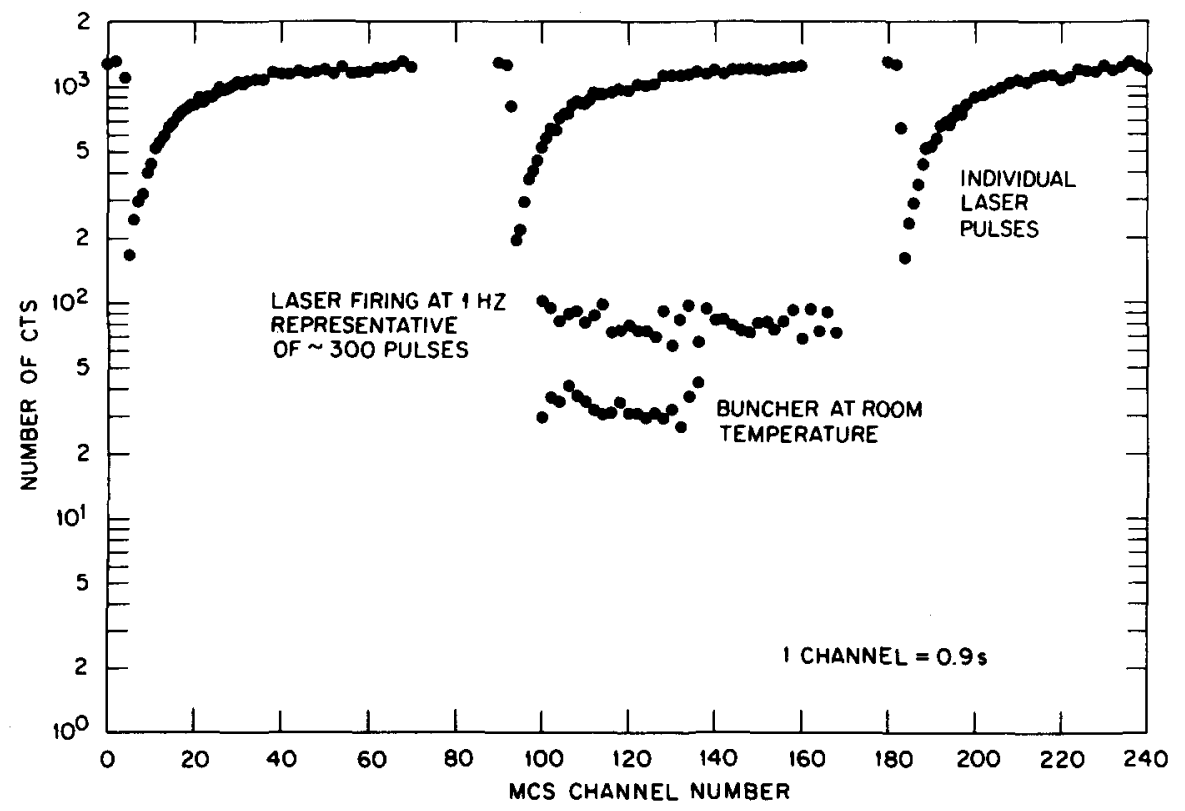

FIG. 7. Experimental tests of the atom buncher. A small Geiger-Mueller counter was used to view the ${ }^{85} \mathrm{Kr}$ atoms remaining on the cold tip as a function of time ( $=0.9 \mathrm{sec} / \mathrm{channel}$ ). Each laser pulse causes nearly all of the atoms to leave the cold surface, as shown in the top portion of the figure. See text for additional interpretation of this figure. 
An apparatus was built to test the atom buncher with krypton. It consisted of a vacuum chamber with a window for the laser to pass through, an Amperex model $18550 \mathrm{Gei}-$ ger-Mueller (G-M) tube, and a source of $4 \%$ enriched radioactive ${ }^{85} \mathrm{Kr}$ gas. A getter pump for impurities other than noble gases was installed so that the system could operate without additional pumping for the duration of the tests. The G-M tube was positioned as close to the cryotip as possible in order to monitor the count rate on the surface without interferring with the heating laser. A multichannel scaler was used to display the count rate as a function of time.

Figure 7 illustrates representative data obtained from the tests. The top graph shows that the atoms do come off of the surface during each individual laser pulse and return to the surface with a recurrence time on the order of $10 \mathrm{sec}$. The center graph illustrates what happens when the laser irradiated the surface at a $1-\mathrm{Hz}$ repetition rate. The measured count rate is consistent with the recurrence time measured for single laser pulses. This graph also demonstrates that the krypton atoms are sitting on the surface in an area that is being heated by the laser. If the entire cold surface were not being heated to $>100 \mathrm{~K}$, the count rate would steadily increase because all of the atoms would eventually collect (i.e., "hide") on the unheated area. The lower graph represents the background count rate from the G-M tube when the buncher is at room temperature with approximately $1 \times 10^{-5}$ Torr of the ${ }^{85} \mathrm{Kr}$ sample in the cell.

Tests on one of the previous designs led to the belief that part of the cold surface in that design was not being heated by the laser pulse, due to an observation that the count rate no longer dropped when the surface was heated by the laser light. The studies reported herein, using our improved design and a better vacuum system, suggest that the early problem was due to an accumulation of outgassing products (such as $\mathrm{H}_{2} \mathrm{O}$ vapor) on the cold surface. The "ice" that forms traps the krypton, and the heating laser could not supply enough energy to melt the ice to release the trapped gases.

\section{SUMMARY}

An atom buncher, which can dramatically increase the probability that an atom (or gaseous molecule) will be in a given region of space at a prescribed time, has been conceptualized, designed, constructed, and tested. We have proven experimentally that the device works well by using radioactive ${ }^{85} \mathrm{Kr}$ as a test atom.

Applications for laser ionization (RIS) have been described as examples of wider applications. These wider applications could include their use in VUV generation wherein small gas bubbles are created inside a vacuum system. Other conceivable uses could be in nozzle replacements for certain atomic, molecular, or ion beam generation.

\section{ACKNOWLEDGMENTS}

This research was sponsored in part by the Office of Health and Environmental Research, U.S. Department of Energy under contract No. W-7405-eng-26 with the Union Carbide Corporation, and in part by the Swiss Nationale Genossenschaft für die Lagerung radioaktiver Abfalle (NAGRA).

'G. S. Hurst, M. G. Payne, S. D. Kramer, and C. H. Chen, Phys. Today 33, No. 9,24 (1980).

${ }^{2}$ G. S. Hurst, C. H. Chen, S. D. Kramer, M. G. Payne, and R. D. Willis, in Science Underground, edited by Michael Martin Nieto, W. C. Haxton, C. M. Hoffman, E. W. Kolb, V. D. Sandberg, and J. W. Toevs, AIP Conference Proceedings No. 96 (American Institute of Physics, New York, 1983), pp. 96-104.

${ }^{3}$ G. S. Hurst, M. G. Payne, S. D. Kramer, and J. P. Young, Rev. Mod. Phys. 51, 767 (1979).

${ }^{4}$ R. G. Wilmoth and S. S. Fisher, Surf. Sci. 72, 693 (1978).

J. F. Ready, J. Appl. Phys. 36, 462 (1965).

'S. D. Kramer, C. H. Chen, M. G. Payne, G. S. Hurst, B. E. Lehmann, and R. D. Willis, in Laser Spectroscopy, edited by H. P. Weber (Springer, Berlin, 1983), pp. 392-395.

${ }^{7}$ Y. S. Touloukian, R. W. Powell, C. Y. Ho, and P. G. Klemens, Thermophysical Properties of Matter, Vol. 1: Thermal Conductivity (IFI/Plenum, New York-Washington, 1970), pp. 237-244.

${ }^{8}$ Robert A. Powell and William A. Blanpied, Thermal Conductivity of Metals and Alloys at Low Temperatures, National Bureau of Standards Circular 556 (U.S. Government Printing Office, Washington, D.C., 1954). 